\title{
A Method of Plastic Gear Inspection Based on Machine Vision
}

\author{
Jianbiao Ding ${ }^{a}$, Yajun Zhang ${ }^{b^{*}}$, Jian Zhuang ${ }^{c}$ and Ri Pan ${ }^{d}$
}

School of Mechanic and Electronic Engineering, Beijing University of Chemical Technology, Beijing 100029, China.

adingabcdefg@163.com, b*a1169@163.com, vipzhuangjian@163.com, dryan_p2011@126.com

Keywords: Machine Vision, Inspection, Gear, CCD camera.

\begin{abstract}
The defect detection of plastic gear is difficult because of its small size, so a method based on machine vision is proposed to inspect plastic gear accurately and quickly. Digital image of plastic gear is collected by CCD camera, and then the image is preprocessed to generate single pixel edge of gear. Based on this edge, the tooth number, circular pitch and diameters of addendum circle and root circle are measured, which are the basis of testing whether the gear is qualified. The experimental results show that this method satisfies the requirements of on-line inspection.
\end{abstract}

\section{Introduction}

Plastic gear has the characteristics of light weight, low cost, small transmission noise, and is widely used in all kinds of precision instruments and micro electro mechanical products. Defects like teeth missing and tooth chipping may be produced in the molding process of gear, which will lead to the increase of transmission noise, wear and decrease of efficiency. So it is necessary to inspect plastic gear to guarantee the quality of gear. But the traditional artificial detection is time-consuming, laborious and the detection accuracy cannot be guaranteed ${ }^{[1]}$.

As a new nondestructive non-contact measurement technology, machine vision technology has advantages of high speed, high precision and strong anti-interference ability. In this paper, an inspection system based on machine vision is designed to test the quality of gear.

\section{Inspection System Structure}

The inspection system includes hardware and software as shown in Fig.1.

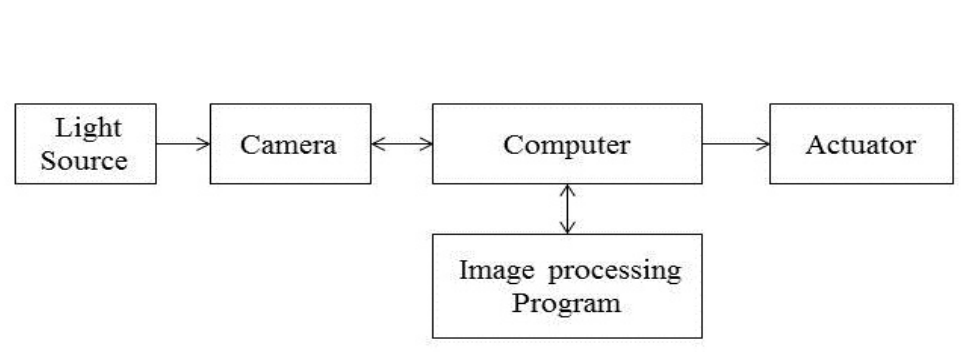

Fig.1 System structure diagram

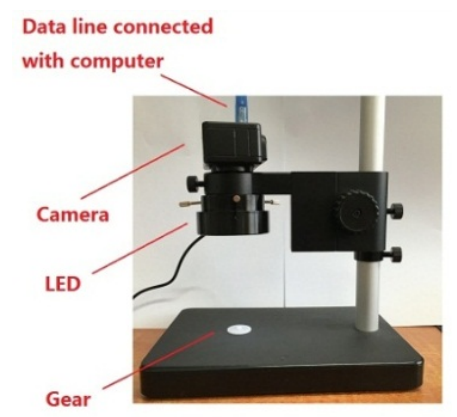

Fig.2 System hardware

The hardware mainly includes CCD camera, light source, holder and computer as shown in Fig.2. The CCD camera is used to collect image, and the light source is used to improve image quality. In this paper, LED ring lamp is used as the light source, and the top illumination is adopted.

The image processing algorithm is programed by HALCON software, which contains image acquisition, gradation, median filtering, threshold segmentation and parameters measurement.

\section{Image Preprocessing}

Image preprocessing includes image filtering and threshold segmentation. Image filtering is used to reduce noise, and threshold segmentation is used to extract gear area from image. 


\subsection{Image Filtering}

Due to the interference of various factors, image will be corrupted by noise inevitably in the process of acquisition and transmission. The median filter is selected to reduce the noise in original image, which de-noises image by replacing the pixel value with median of neighboring pixel values ${ }^{[2]}$. A $3 \times 3$ square median filtering template is adopted in this paper, and the result contains less noise without much loss of edge information as shown in Fig.3 and Fig.4.

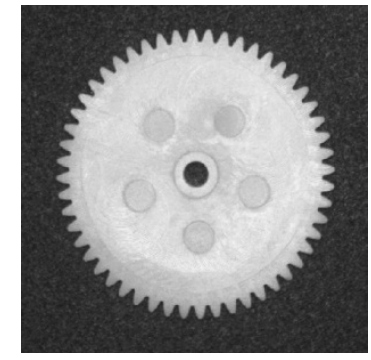

Fig.3 Original image

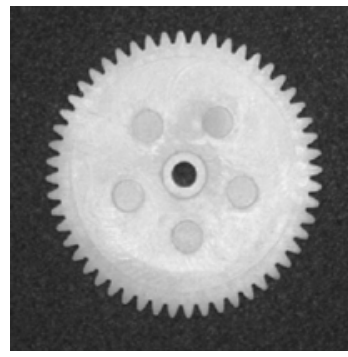

Fig.4 Result of filtering

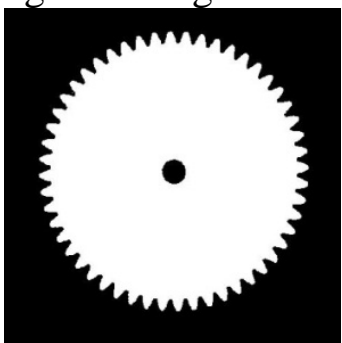

Fig.5 Result of threshold segmentation

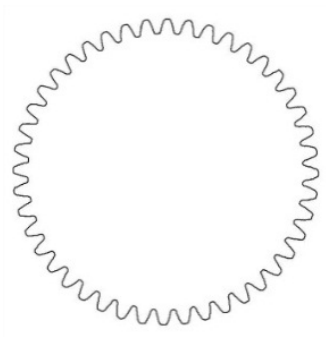

Fig.6 Extracted gear profile

\subsection{Threshold Segmentation}

Threshold segmentation method is an image segmentation technique based on region. According to gray level, pixels are divided into different attributes subsets which correspond to different real scenes. The division is achieved by selecting one or more thresholds from the gray level.

The principle of single threshold segmentation is:

$$
g(x, y)= \begin{cases}0 & f(x, y)<T \\ 255 & f(x, y)>T\end{cases}
$$

Where, $T$ is the threshold, $f(x, y)$ is the gray value of pixel $(x, y)$ before segmentation, $g(x, y)$ is the gray value of pixel $(x, y)$ after segmentation.

The image pixels are set to white (255) and black (0) after threshold segmentation. Fig.5 shows image after segmentation which contains white and black area, and the white area is the extracted gear region. After extracting the gear region, edge detection algorithm is applied to extract the contour of gear which is the basis of subsequent parameters measurement ${ }^{[3]}$ as shown in Fig.6.

\section{Parameters Measurement}

Based on the optimized gear tooth profile obtained by image preprocessing, the main shape parameters of gear are measured. To get the centers of addendum circle and root circle, the center of gear needs to be located before parameters measurement.

\subsection{Locating the Center of Gear}

To locate the center accurately and quickly, multiple methods including centroid method, addendum circle fitting and root circle fitting are used to obtain center coordinates. The average value of center coordinates calculated by these three methods is regarded as final center coordinate.

$$
(x, y)=\left(\frac{x_{1}+x_{2}+x_{3}}{3}, \frac{y_{1}+y_{2}+y_{3}}{3}\right)
$$

Where, $(x, y)$ is the final center coordinate, $\left(x_{1}, y_{1}\right) 、\left(x_{2}, y_{2}\right) 、\left(x_{3}, y_{3}\right)$ are center coordinates calculated by centroid method, addendum circle fitting and root circle fitting, respectively.

The principle of centroid method is to calculate the average value of coordinates of all pixels in the gear region. This average value is regarded as the center coordinate. Considering that the shaft hole of gear will influence the calculating of center, white pixels are used to fill the shaft hole to gain a whole gear region. Fig.7 shows the gear region without shaft hole. 


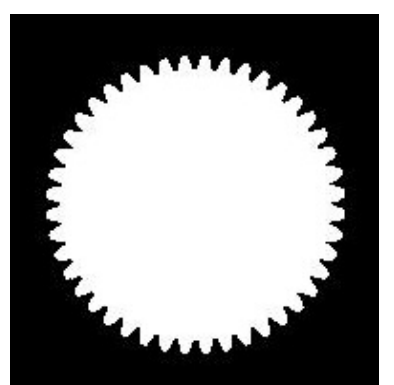

Fig.7 Gear region

without shaft hole

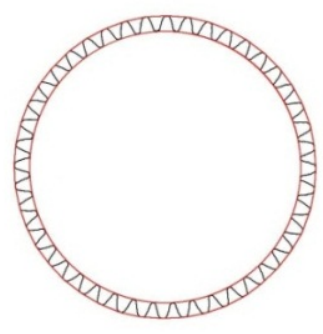

Fig.8 Fitness of addendum circle and root circle

\subsection{Measurement of Diameters of Addendum Circle and Root Circle}

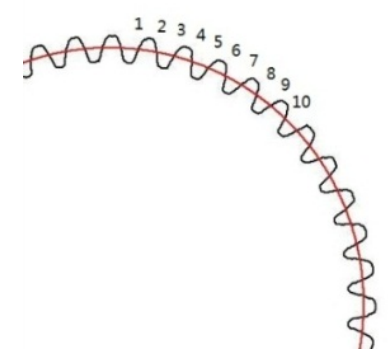

Fig.9 Measurement of circular pitch

The diameters of addendum circle and root circle are measured by the following steps:

1) Since the extracted gear profile cannot be perfect circle, shape_trans (an operator of HALCON software) operator is used to fit smallest external polygon of the gear profile, and then fit_circle_contour_xld operator is used to fit minimum circumcircle of the smallest external polygon. The minimum circumcircle is regarded as addendum circle which leads to the obtainment of center $\left(x_{1}, y_{1}\right)$ and diameter $R_{1}$ of addendum circle.

2) shape_transoperator is used to fit maximum incircle of the gear profile. Using the maximum incircle to calculate the center $\left(x_{2}, y_{2}\right)$ and diameter $R_{2}$ of root circle. Fig.8 shows the results of fitness of addendum circle and root circle.

The diameter of pitch circle $R_{3}$ is:

$$
R_{3}=\frac{\left(5 * R_{1}+4 * R_{2}\right)}{9}
$$

The diameter of pitch circle is obtained to be compared with the theoretical diameter $22 \mathrm{~mm}$ to calculate the measurement error of system. The measurement error $E$ is calculated as:

$$
E=\frac{R_{3}-22}{22}
$$

\subsection{Measurement of Circular Pitch and Tooth Number}

The measurement of circular pitch is achieved by drawing pitch circle whose diameter is $22 \mathrm{~mm}$ and center is the final coordinate obtained in section 4.1. The pitch circle intersects gear profile at different points as shown in Fig.9, and these points are defined as 1, 2, 3, 4...n. The arc length between two adjacent teeth on the pitch circle is circular pitch ${ }^{[4,5]}$, so the circular pitches are $l_{13}, l_{24}$, $l_{35} \ldots . . . m$. The tooth number is half of the intersection points of pitch circle and gear profile.

\section{Experimental Results and Discussion}

Plastic gears with modulus of 0.5 and tooth number of 44 are used as the experimental object. Its diameter of addendum circle is $23 \mathrm{~mm}$, diameter of pitch circle is $22 \mathrm{~mm}$, and theoretical tooth pitch is $1.571 \mathrm{~mm}$.According to national standard, single pitch deviation of precision level ten is $28 \mathrm{um}$.

Hundreds pictures of qualified gear and defective gear are collected as experiment pictures. Table 1 shows part of the experimental results, number 1 to 7 are qualified gears, and number 8 to 10 are gears with teeth missing. According to these results, the measurement error $E$ is no more than $1 \%$, and the detection error is mainly caused by the calibration error and optical error ${ }^{[6]}$.The tooth numbers and maximum pitches of 7th to 10th gears differ greatly from the theoretical value, which means these gears are defect gears. The whole experimental results show that the inspection system can effectively detect defect gears with $99 \%$ accuracy rate and $0.3 s$ measuring time. 
Table 1 Experimental results of parameters measurement

\begin{tabular}{ccccccccc}
\hline $\begin{array}{c}\text { Series } \\
\text { No. }\end{array}$ & $\begin{array}{c}\text { Tooth } \\
\text { Number }\end{array}$ & $R_{1}(\mathrm{~mm})$ & $R_{2}(\mathrm{~mm})$ & $R_{3}(\mathrm{~mm})$ & $E$ & $\begin{array}{c}\text { Maximum } \\
\text { Pitch(mm) }\end{array}$ & $\begin{array}{c}\text { Minimum } \\
\text { Pitch(mm) }\end{array}$ & $\begin{array}{c}\text { Mean Pitch } \\
(\mathrm{mm})\end{array}$ \\
\hline 1 & 44 & 23.0408 & 20.7681 & 22.03071 & $0.14 \%$ & 1.59681 & 1.54495 & 1.57 \\
2 & 44 & 23.0434 & 20.7681 & 22.03216 & $0.15 \%$ & 1.58304 & 1.54956 & 1.57 \\
3 & 44 & 23.0214 & 20.7321 & 22.00393 & $0.02 \%$ & 1.57114 & 1.54172 & 1.57 \\
4 & 44 & 23.0204 & 20.7321 & 22.00338 & $0.02 \%$ & 1.57912 & 1.54605 & 1.57 \\
5 & 44 & 23.04 & 20.7331 & 22.01471 & $0.06 \%$ & 1.57715 & 1.55041 & 1.57 \\
6 & 44 & 22.9319 & 20.6197 & 21.90426 & $0.44 \%$ & 1.58667 & 1.54575 & 1.56 \\
7 & 44 & 22.9345 & 20.6197 & 21.9057 & $0.43 \%$ & 1.59398 & 1.55011 & 1.56 \\
8 & $\underline{43}$ & 22.9982 & 20.5101 & 21.89238 & $0.49 \%$ & $\underline{3.14535}$ & 1.54243 & 1.57 \\
9 & $\underline{43}$ & 22.9815 & 20.5372 & 21.89514 & $0.48 \%$ & $\underline{3.11973}$ & 1.54751 & 1.57 \\
10 & $\underline{43}$ & 22.9026 & 20.5743 & 21.8678 & $0.60 \%$ & $\underline{3.13614}$ & 1.5451 & 1.57 \\
\hline
\end{tabular}

\section{Summary}

A gear inspection system based on machine vision is designed to reject unqualified gear in this paper. CCD camera and LED ring lamp are used to get high quality image. HALCON program is used to process image to measure gear parameters which are basis for testing gear. Measurement experiments of hundreds pictures of qualified gear and defective gear are carried out. The results show that the system's detection time is less than $0.3 s$, measurement error is less than $1 \%$, and the accuracy rate reaches $99 \%$, which means rapid on-line detection of plastic gear has been realized.

\section{Acknowledgement}

This work was financially supported by the Fundamental Research Funds for the Central Universities (YS1403, ZY1521); the China Postdoctoral Science Foundation (2015M570920); State Key Laboratory of Organic-Inorganic Composites (Open issue 201601011); Beijing Natural Science Foundation (2131003).

\section{References}

[1]. Gadelmawla E.S. Computer vision algorithms for measurement and inspection of spur gears [J]. Measurement. Vol. 44 (2011) No. 9, p. 1669-1678.

[2]. Meher S K, Singhawat B. An Improved Recursive and Adaptive Median Filter for High Density Impulse Noise[J]. AEU - International Journal of Electronics and Communications. Vol. 68(2014) No. 12, p. 1173-1179.

[3]. Biswas R, Sil J. An Improved Canny Edge Detection Algorithm Based on Type-2 Fuzzy Sets[J]. Procedia Technology. Vol. 4 (2012) No. 11, p. 820-824.

[4]. Lin C, Cao X J, Fan Y, et al. Pitch deviation measurement and analysis of curve-face gear pair [J]. Measurement. Vol. 81 (2016), p. 95-101.

[5]. Zhang M, Shi Z Y, Mathieu L, et al. Geometric Product Specification of Gears: The GeoSpelling Perspective[J]. Procedia Cirp. Vol. 27(2015) No. 4, p. 90-96.

[6]. Sammartinia M P, Chiffre L D. Development and validation of a new reference cylindrical gear for pitch measurement [J]. Precision Engineering. Vol. 24 (2000) No. 4, p. 302-309. 